\title{
Use of individual retention modeling for gradient optimization in hydrophilic interaction chromatography: Separation of nucleobases and nucleosides
}

\author{
Eva Tyteca $^{\mathrm{a}, *}$, Davy Guillarme ${ }^{\mathrm{b}}$, Gert Desmet ${ }^{\mathrm{a}}$ \\ a Vrije Universiteit Brussel, Department of Chemical Engineering, Pleinlaan 2, B-1050 Brussels, Belgium

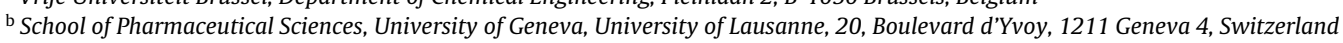

\section{A R T I C L E I N F O}

\section{Article history:}

Received 7 August 2014

Received in revised form

24 September 2014

Accepted 25 September 2014

Available online 13 October 2014

\section{Keywords:}

Computer-assisted method development

Individual retention modeling

Nucleobases

Nucleosides

HILIC

\begin{abstract}
A B S T R A C T
In this study, the separation of twelve nucleobases and nucleosides was optimized via chromatogram simulation (i.e., prediction of individual retention times and estimation of the peak widths) with the use of an empirical (reversed-phase) non-linear model proposed by Neue and Kuss. Retention time prediction errors of less than $2 \%$ were observed for all compounds on different stationary phases. As a single HILIC column could not resolve all peaks, the modeling was extended to coupled-column systems (with different stationary phase chemistries) to increase the separation efficiency and selectivity. The analytical expressions for the gradient retention factor on a coupled column system were derived and accurate retention time predictions were obtained ( $<2 \%$ prediction errors in general). The optimized gradient (predicted by the optimization software) included coupling of an amide and an pentahydroxy functionalized silica stationary phases with a gradient profile from 95 to 85\%ACN in $6 \mathrm{~min}$ and resulted in almost baseline separation of the twelve nucleobases and nucleosides in less than $7 \mathrm{~min}$. The final separation was obtained in less than $4 \mathrm{~h}$ of instrument time (including equilibration times) and was fully obtained via computer-based optimization. As such, this study provides an example of a case where individual retention modeling can be used as a way to optimize the gradient conditions in the HILIC mode using a non-linear model such as the Neue and Kuss model.
\end{abstract}

(c) 2014 Elsevier B.V. All rights reserved.

\section{Introduction}

To speed up the method development (MD) process in chromatography, fully or semi-automated MD software programs have been developed for reversed phase liquid chromatography (RPLC) [1-6]. These automated MD strategies for RPLC described in literature are either search-based (e.g. using the Simplex method) [7], model-based (e.g. Drylab [8], Chromsword) [9] or based on a combination of both (design of experiments (DoE), multiple linear regression (MLR) [10], predictive elution window stretching and shifting method (PEWS ${ }^{2}$ ) [11]). Recently, the PEWS ${ }^{2}$ method was successfully applied for the gradient optimization in hydrophilic interaction chromatography (HILIC) [12].

HILIC is becoming more and more popular, for the determination of hydrophilic compounds, poorly retained in RPLC conditions, and for the analysis of ionizable compounds [13]. HILIC retention can be

\footnotetext{
* Corresponding author. Tel.: +32 22693617.

E-mail address: eva.tyteca@vub.ac.be (E. Tyteca).
}

considered as a mixed-mode mechanism, combining hydrophilic partitioning of the analytes between the organic-rich mobile phase and the water enriched layer partially immobilized on the stationary phase, compounds adsorption through hydrogen bonds, and electrostatic and ionic interactions [14]. Although both the linear reversed phase (semi-log) (Eq. (1)) and the normal phase (log-log) retention relationships have been used in literature to model HILIC retention [15], the dependency of $\ln (k)$ on both $\varphi$ and $\log (\varphi)$ in the HILIC separation mode does not follow a strict linear relationship $[15,16]$. Based on these observations, Jin et al. proposed a mixed model combining partitioning and adsorption terms (Eq. (2)) to describe the retention behavior in HILIC [15]. Jandera and Hájek [17] introduced an extended model including one extra parameter to better describe the retention at low percentages of water. Other (reversed-phase) non-linear models such as the quadratic model and the empirical model from Neue and Kuss (Eq. (3)) have also been proposed in literature to describe the retention relationship in the HILIC separation mode [12].

$\ln (k)=\ln \left(k_{w}\right)-S \varphi$ 
$\ln (k)=\ln \left(k_{w}\right)+S_{1} \varphi+S_{2} \ln (\varphi)$

$\ln (k)=\ln \left(k_{w}\right)+2 \ln \left(1+S_{2} \varphi\right)-\frac{S_{1} \varphi}{1+S_{2} \varphi}$

where $\varphi$ is the fraction of water, $k_{w}$ is the extrapolated values of $k$ for $\varphi=0$ (i.e., pure ACN) and $S$ is the solvent strength parameter which is a constant for a given compound and organic solvent [18,19], $S_{1}$ the slope for the non-linear models, $S_{2}$ the curvature coefficient [21].

Greco et al. reported determination coefficients $R^{2}$ above 0.99 for 14 benzoic acids using Eq. (2) [14]. In a recent study, including several analytes possessing diverse physico-chemical properties, we reported $R_{\text {adjusted }}^{2}$ and $Q^{2}$-values for the empirical Neue and Kuss (Eq. (3)) model, close to those of the mixed model (Eq. (2)) [12]. However, the gradient retention predictions were much less accurate in HILIC than RPLC, restricting the use of commercial software packages requiring the simulation of the retention of every peak in the chromatogram [12].

The expression for the gradient retention factor can be found by solving the fundamental gradient equation:

$t_{0}=\int_{0}^{t_{R}-t_{0}} \frac{d t_{s}}{k(\varphi)}$

where $t_{R}$ and $t_{0}$ are the total retention time and the column dead time, respectively [20] and $t_{\mathrm{s}}$ is the time in the stationary phase. Whereas the mixed model (Eq. (2)) no longer has an analytical solution to the fundamental gradient equation, it is one of the virtues of the Neue and Kuss-model (Eq. (3)) that it is easily amenable to an analytical solution, leading to the following expression for the effective retention factor $k_{\text {eff }}=\left(t_{R}-t_{0}\right) / t_{0}[21]$ :
95\%ACN for eight nucleosides on six different columns, using Eq. (2). However, no gradient predictions were ever made using this mixed model. Pappa-Louisi and coworkers reported on the gradient retention time predictions of amino acids in HILIC using the LSSmodel (Eq. (1)) for linear, multi-linear and curved elution profiles with the same starting concentration.

\section{Experimental}

Water was obtained from a Milli-Q water purification system from Millipore (Bedford, MA, USA). Acetonitrile (ACN), methanol $(\mathrm{MeOH})$ and formic acid were of ULC-MS grade and purchased from Biosolve (Valkenswaald, Netherlands). Ammonium acetate ( $>98 \%$, Sigma-Aldrich, Belgium) buffer $10 \mathrm{mM}$ was prepared by weighing adequate mass of ammonium acetate. The $\mathrm{pH}$ was adjusted to 3.0 or 6.0 with formic acid (FA). All compounds (thymine, uridine, inosine, adenine, uracil, hypoxanthine, cytidine, thymidine, adenosine, cytosine, guanine and guanosine) were purchased from Sigma-Aldrich. Stock solutions of $1000 \mathrm{ppm}$ were prepared in $\mathrm{MeOH}$ except for guanosine and guanine $(0.1 \% \mathrm{FA}$ and $\mathrm{KOH}$ solutions, respectively). The samples were diluted in $\mathrm{ACN}+0.1 \%$ FA and injected at $100 \mathrm{ppm}$. The injection volume was $1 \mu \mathrm{L}$. All experiments were performed on an Agilent Infinity 1290 system with a dwell volume of $112 \mu \mathrm{L}$. The columns were Supelco HILIC and Supelco OH5, i.e., bare silica and pentahydroxy functionalized silica respectively $(100 \mathrm{~mm} \times 2.1 \mathrm{~mm}, 2.7 \mu \mathrm{m})$, and Waters Acquity Amide, i.e., amide functionalized silica $(100 \mathrm{~mm} \times 2.1 \mathrm{~mm}$, $1.7 \mu \mathrm{m})$. The dead times were measured at 70\%ACN: 0.419, 0.381 and $0.422 \mathrm{~min}$ on the bare silica, the pentahydroxy and the amide stationary phase, respectively. The flow rate was $0.5 \mathrm{~mL} / \mathrm{min}$.

$k_{\text {eff }}=\frac{t_{D}}{t_{0}}+\frac{\left[\phi_{0}+\left(1+S_{2} \phi_{0}\right) / S_{1} \ln \left(1+\beta k_{w}^{\prime} S_{1}\left(t_{0}-t_{D} / k_{0}\right) \exp \left(-S_{1} \phi_{0} / 1+S_{2} \phi_{0}\right)\right)\right] /\left[1-\left(S_{2}\left(1+S_{2} \phi_{0}\right)\right) / S_{1} \ln \left(1+\beta k_{w}^{\prime} S_{1}\left(t_{0}-t_{D} / k_{0}\right) \exp \left(-S_{1} \phi_{0} / 1+S_{2} \phi_{0}\right)\right)\right]-\phi_{0}}{\beta t_{0}}$

where $t_{D}$ is the dwell time, $\beta$ is the gradient slope, defined as $\left(\varphi_{e}-\varphi_{0}\right) / t_{G}$ and $k_{0}$ is the isocratic retention factor at the start of the gradient, i.e. for $\varphi=\varphi_{0}$.

In a previous study, involving samples with pteridines, sugars and a drug mixture of venlafaxine, tramadol and their metabolites in HILIC we observed strongly non-linear retention relationships, that were so complex that they could not be modeled using any of the existing non-linear retention models. As a consequence, the traditional retention modeling approach [22] that is so successful and widespread used in RPLC, could not be applied and we had to switch to a model-guided search approach (so-called PEWS ${ }^{2}$ technique $[11,12])$. For a sample containing compounds behaving more "nicely", individual retention modeling using a non-linear 3parameter model (mixed model and the empirical model from Neue and Kuss) could be used to optimize the gradient conditions.

In the present contribution, we report on the optimization of a HILIC separation of twelve nucleobases and nucleosides using this individual retention modeling and the coupling of columns (with different stationary phases). In the adopted MD work flow, three different column chemistries and two mobile phase $\mathrm{pH}$ were tested.

The separation of nucleic acids and analogs are of great interest in pharmaceutical sciences, genomics, genetics and others [23]. Massolini and coworkers used narrow bore columns with gradient elution (ZIC-HILIC, $150 \mathrm{~mm} \times 2.1 \mathrm{~mm}, 5 \mu \mathrm{m}$ ) to separate the 12 nucleobases and nucleosides included in this study in $55 \mathrm{~min}$. Nikitas et al. used reversed-phase liquid chromatography (without ion paring reagent) to separate 16 nucleobases and nucleosides using two-segment gradient profiles [24]. The linear log-log retention relationship showed better prediction accuracy compared to the semi-log retention relationship (Eq. (1)), both for simple linear and multi-linear gradient profiles. Jin et al. [15] reported $R^{2}$-values around 0.999 and isocratic prediction errors smaller than $5 \%$ at
Temperature was set $25^{\circ} \mathrm{C}$. UV-detection was set at $254 \mathrm{~nm}$. The isocratic scouting runs were performed using different subgroups (see different colors in Fig. 3), to avoid overlapping peaks and ensure accurate retention time determination.

\subsection{Data analysis}

Matlab software (2009) was used for the estimation of the model parameters and the calculation of the optimal gradient conditions via an in-house written Matlab code. The retention factors of the isocratic scouting runs were used to obtain the model parameters (Eqs. (2) and (3)) via least squares fitting using the Matlab ${ }^{\circledR}$ routine lsqcurvefit. Gradient retention times were either predicted by implementing the analytical expression (Eq. (5), for the Neue-Kuss model) in Matlab-software or, for the other non-linear model (Eq. (2)), via numerical integration of the fundamental gradient equation via an in-house written Matlab routine based on the trapezoid rule.

$$
\begin{aligned}
t_{0} & =\int_{0}^{t_{R}-t_{0}} \frac{d t_{s}}{k(\varphi)}=\frac{t_{D}}{k_{0}}+\frac{1}{\beta} \int_{\varphi_{0}}^{\varphi_{\text {elution }}} \frac{d \varphi}{k(\varphi)} \Leftrightarrow \beta \cdot\left(t_{0}-\frac{t_{D}}{k_{0}}\right) \\
& =\int_{\varphi_{0}}^{\varphi_{\text {elution }}} \frac{d \varphi}{k(\varphi)}=I
\end{aligned}
$$

The percentage of ACN at elution $\varphi_{\text {elution }}$ is obtained by minimizing $I-\beta^{*}\left(t_{0}-t_{D} / k_{0}\right)$. From $\varphi_{\text {elution }}$, the effective retention factor $k_{\text {eff }}$ can be calculated via:

$k_{\text {eff }}=\frac{t_{R}-t_{0}}{t_{0}}=\frac{t_{D}}{t_{0}}+\frac{\phi_{\text {elution }}-\phi_{0}}{\beta t_{0}}$ 
Table 1

$R_{\text {adjusted }}^{2}$ and $Q^{2}$ values and the retention parameters $k_{w}, S_{1}$ and $S_{2}$ for the 12 nucleobases and nucleosides on the pentahydroxy stationary phase column pH $=6$.

\begin{tabular}{|c|c|c|c|c|c|c|c|c|c|c|}
\hline & \multicolumn{5}{|l|}{ Eq. (3) } & \multicolumn{5}{|l|}{ Eq. (2) } \\
\hline & $R^{2}$ & $Q^{2}$ & $\ln \left(k_{w}\right)$ & $S_{1}$ & $S_{2}$ & $R^{2}$ & $Q^{2}$ & $\ln \left(k_{w}\right)$ & $S_{1}$ & $S_{2}$ \\
\hline Thymine & 1.0000 & 0.9998 & 1.96 & 21.2 & 2.4 & 0.9988 & 0.9911 & -0.45 & -3.3 & -0.6 \\
\hline Uracil & 0.9998 & 0.9996 & 3.12 & 39.3 & 4.2 & 0.9989 & 0.9922 & -1.54 & -1.0 & -1.2 \\
\hline Thymidine & 0.9991 & 0.9935 & 2.88 & 31.7 & 3.3 & 0.9993 & 0.9985 & -0.95 & -2.7 & -1.0 \\
\hline Uridine & 1.0000 & 1.0000 & 3.09 & 42.4 & 4.7 & 0.9989 & 0.9918 & -1.84 & -0.3 & -1.2 \\
\hline Inosine & 0.9999 & 0.9991 & 3.66 & 36.7 & 3.5 & 0.9994 & 0.9960 & -0.85 & -2.9 & -1.2 \\
\hline Adenosine & 1.0000 & 0.9999 & 3.93 & 50.0 & 5.0 & 0.9990 & 0.9925 & -1.93 & -0.3 & -1.5 \\
\hline Hypoxanthine & 0.9999 & 0.9997 & 4.30 & 44.0 & 4.0 & 0.9992 & 0.9938 & -1.14 & -2.4 & -1.4 \\
\hline Cytosine & 0.9994 & 0.9968 & 1.96 & 21.2 & 2.4 & 0.9998 & 0.9991 & -0.45 & -3.3 & -0.6 \\
\hline Adenine & 1.0000 & 0.9999 & 0.93 & 18.5 & 2.7 & 0.9990 & 0.9929 & -1.11 & -1.5 & -0.5 \\
\hline Cytidine & 0.9999 & 0.9992 & 1.36 & 21.7 & 2.7 & 0.9994 & 0.9958 & -1.12 & -2.4 & -0.6 \\
\hline Guanine & 0.9994 & 0.9984 & 5.08 & 72.0 & 6.4 & 0.9993 & 0.9987 & -2.27 & -0.6 & -1.7 \\
\hline Guanosine & 0.9994 & 0.9986 & 5.23 & 62.9 & 5.3 & 0.9989 & 0.9979 & -1.43 & -2.9 & -1.6 \\
\hline
\end{tabular}

The prediction errors were calculated as $\left(t_{R, \text { experiment }}-\right.$ $\left.t_{R, \text { predicted }}\right) / t_{R, \text { experiment }}$ with $t_{R}=t_{0} *\left(1+k_{\text {eff }}\right)$.

The gradient optimization strategy consists of a grid search, scanning through all combinations of $\varphi_{0}$ and $\beta(100 \times 100$ conditions with $\ln (\beta)$ equally spaced between 0.001 and 0.5 and $\varphi_{0}$ between 0.05 and 0.35 ). For all compounds, the retention time $t_{R}$ is predicted and the resolution $R_{S}$ (Eq. (8)) estimated under all the combinations of $\varphi_{0}$ and $\beta$.

$R_{S}=\frac{\sqrt{N}}{4} \frac{\Delta k}{1+k_{\text {elution }}}$

where $k_{\text {elution }}$ is the average retention factor of the two compounds when eluting from the column, i.e. for $\varphi=\varphi_{\text {elution }}$. The best gradient conditions correspond to baseline separation $\left(R_{s, \text { crit }}=1.6\right)$ within the shortest possible analysis time $\left(t_{R, \text { analysis }}=t_{R, \text { last compound }}\right)$. When multiple good solutions are found, these solutions are ranked, according to their respective analysis times.

For the coupled columns with different stationary phases, the same approach can be followed, using the analytical expressions for a coupled column system (Eqs. (9)-(12)). In Eq. (8) the isocratic plate number $N$ is the sum of the individual plate numbers $\left(N_{\text {column } 1}+N_{\text {column } 2}\right)$ and $k_{\text {elution }}$ is the retention factor at elution from the second column.
Note that other quality criterions, such as the $S$ criterion [25], could be used in the optimization.

\section{Results and discussion}

\subsection{Retention modeling}

In a first step, the isocratic retention relationships for the twelve nucleobases and nucleosides in HILIC mode were established by performing 10 isocratic measurements $(95,94,93,92,91,90,88$, $85,80$ and $70 \% A C N)$, on three different stationary phase chemistries at two different $\mathrm{pH}$-values. Subsequently, we performed a least squares fitting of the obtained isocratic retention factors, using Eqs. (2) and (3), to estimate the retention parameters. The high number of isocratic runs was based on our previous experience with pteridines, sugars and the drug mixture of venlafaxine, tramadol and their metabolites, showing that very strong complex non-linearities can occur in HILIC.

In Fig. 1, the established models for all compounds on two stationary phase chemistries are given $(\mathrm{pH}=6)$. The bare silica showed less retention for all compounds compared to the pentahydroxy column. Both retention models (Eqs. (2) and (3), resp. red and blue curves in Fig. 1) were able to describe the isocratic retention data

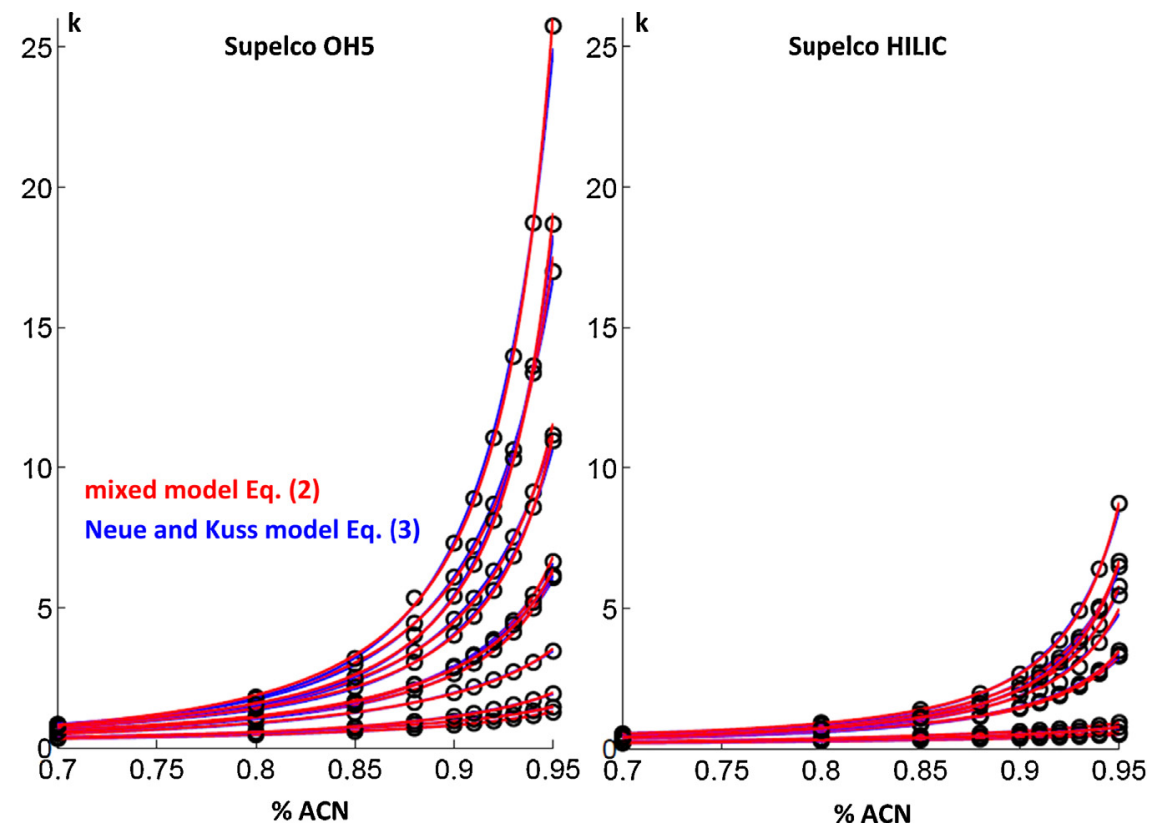

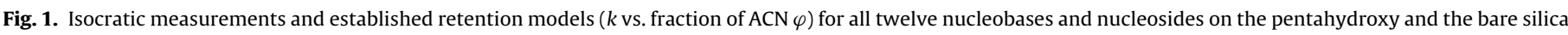
stationary phases. (For interpretation of the references to color in text, the reader is referred to the web version of the article.) 
Table 2

Average gradient prediction errors for 12 nucleobases and nucleosides and four gradient runs (95-85\%ACN in 2 and $6 \mathrm{~min}, 92-85 \% \mathrm{ACN}$ in 2 and $6 \mathrm{~min}$ ).

\begin{tabular}{llll}
\hline & & Eq. (2) & Eq. (3) \\
\hline Pentahydroxy & pH 3 & $2.8 \%$ & $3.0 \%$ \\
& pH 6 & $3.0 \%$ & $3.5 \%$ \\
\multirow{2}{*}{ Bare silica } & pH 3 & $3.6 \%$ & $3.2 \%$ \\
& pH 6 & $2.1 \%$ & $1.8 \%$ \\
Amide & pH 6 & $3.5 \%$ & $3.6 \%$ \\
\hline
\end{tabular}

very accurately and the difference between both retention models was very small (Fig. 1). In Table 1 , the $R_{\text {adjusted }}^{2}$ and $Q^{2}$ values and the obtained retention parameters are provided for all compounds on the pentahydroxy column.

Since using all ten isocratic runs to establish the retention models for gradient optimization purposes would be too much work in a practical application, we investigated whether the retention models could also be established using a smaller number of scouting runs. For this purpose, four scouting isocratic runs $(95,90,85$ and $80 \% \mathrm{ACN}$ ) were used to investigate the possibilities of gradient retention time modeling. Subsequently, the retention model parameters obtained from these isocratic runs were used to predict the retention under four different gradients with various gradient times $t_{G}$ and different starting compositions $\varphi_{0}$ (95-85\%ACN in 2 and $6 \mathrm{~min}, 92-85 \% \mathrm{ACN}$ in 2 and $6 \mathrm{~min}$ ). The experimental gradient retention factors were compared with the predicted ones and the percentage errors were calculated.

All absolute gradient predictions errors were smaller than $5 \%$ with an average of around $3 \%$ for all $\mathrm{pHs}$ and columns (see Table 2 ). No significant difference in prediction errors was observed between the different gradient runs and between the mixed model and the empirical model from Neue and Kuss. Fig. 2 shows the correlation plot for $k_{\text {predicted }}$ vs. $k_{\text {experiment }}$ using these four isocratic scouting runs.

\subsection{Separation optimization}

\subsubsection{Individual columns}

As shown in Section 3.1 both the empirical Neue and Kuss-model and the mixed model gave similar gradient prediction errors. However, since the calculation time using the analytical expression (Eq.

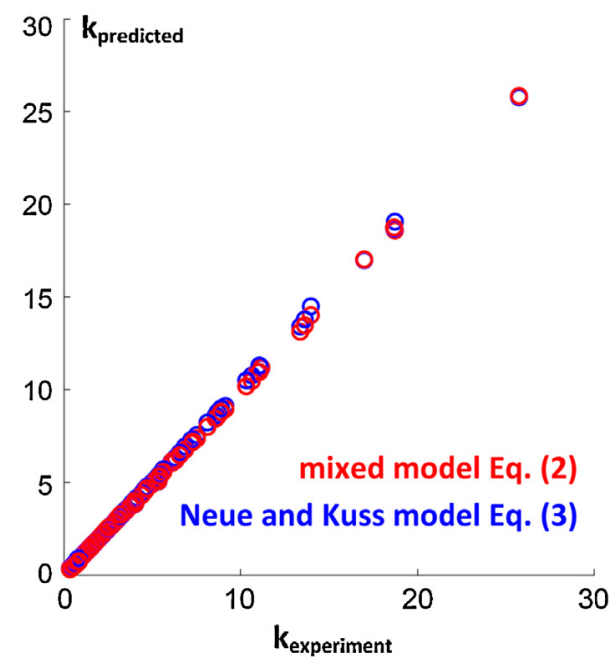

Fig. 2. Correlation plot for $k_{\text {predicted }}$ vs. $k_{\text {experiment }}$ for 12 nucleobases and nucleosides including four different gradients (95-85\%ACN in 2 and $6 \mathrm{~min}, 92-85 \% \mathrm{ACN}$ in 2 and $6 \mathrm{~min}$ ) on the pentahydroxy stationary phase at $\mathrm{pH}=6$. Retention parameters obtained using four isocratic scouting runs $(95,90,85$ and $80 \% \mathrm{ACN})$.
Table 3

Retention time predictions $(\mathrm{min}$ ) on the pentahydroxy stationary phase at $\mathrm{pH}=6$. Gradient conditions: $94-90.6 \%$ ACN in 3.36 min.

\begin{tabular}{lllll}
\hline & $\begin{array}{l}t_{R, \text { predicted }} \\
(\mathrm{min})\end{array}$ & $\begin{array}{l}t_{R, \text { experiment }} \\
(\mathrm{min})\end{array}$ & $\begin{array}{l}\text { \%error } \\
\text { (Eq. (3)) }\end{array}$ & $\begin{array}{l}\text { \%error } \\
\text { (Eq. (1)) }\end{array}$ \\
\hline Thymine & 0.819 & 0.819 & $-0.02 \%$ & $-3.80 \%$ \\
Uracil & 0.881 & 0.874 & $0.79 \%$ & $-2.70 \%$ \\
Thymidine & 1.024 & 1.024 & $0.03 \%$ & $-4.40 \%$ \\
Uridine & 1.497 & 1.480 & $1.17 \%$ & $-3.30 \%$ \\
Inosine & 2.067 & 2.069 & $-0.11 \%$ & $-7.30 \%$ \\
Adenosine & 2.147 & 2.130 & $0.79 \%$ & $-5.00 \%$ \\
Hypoxanthine & 2.212 & 2.200 & $0.54 \%$ & $-6.10 \%$ \\
Cytosine & 2.962 & 2.987 & $-0.02 \%$ & $-3.80 \%$ \\
Adenine & 3.151 & 3.119 & $0.79 \%$ & $-2.70 \%$ \\
Cytidine & 3.967 & 3.931 & $0.03 \%$ & $-4.40 \%$ \\
Guanine & 3.828 & 3.891 & $-1.62 \%$ & $-3.56 \%$ \\
Guanosine & 4.627 & 4.655 & $-0.60 \%$ & $0.60 \%$ \\
\hline
\end{tabular}

(8)) via the Neue and Kuss isocratic retention relationship is significantly reduced compared to the numerical integration via the mixed model, gradient optimization was performed with the Neue and Kuss retention parameters. Because the modeling showed to be reasonably accurate (consistently $<5 \%$ ), all peaks were individually modeled in the MD strategy. This is an alternative gradient optimization approach compared to the PEWS ${ }^{2}$ strategy which has already been successfully applied for the gradient optimization in the HILIC mode [12], when the retention mechanism of the compounds is too complex to be accurately described by the simple model from Neue and Kuss. First the retention time and peak width of every peak in the mixture is simulated under various gradient conditions. Then, the corresponding resolution map $R_{S}$ (Eq. (8)) is constructed from which the optimal gradient conditions can be obtained. The optimal condition corresponds to the case where the critical resolution $R_{S, \text { crit }}$ was maximal, similar to the resolution maps constructed in commercial RPLC modeling software based on the linear solvent strength (LSS) model (Eq. (1)). As an example, the best possible separation on the pentahydroxy stationary phase is provided in Fig. 3. The relatively high concentrations (100 ppm), common for UV-detection, and an injected volume of $1 \mu \mathrm{L}$ resulted in good chromatograms with a high signal-to-noise ratio. As the injected mass (100 ng) was quite low, no problems of overloading occurred. Moreover, since there is no irreversible adsorption of the compounds at the surface of the stationary phase, the retention times were reliable and identical for both the individual compounds and the mixture compounds. Note that these concentrations are not critical for the estimation of the retention model parameters. In case more expensive or scarce compounds would be used, lower concentrations could be obviously considered during the scouting runs. Accurate retention time predictions (maximal error of $1.6 \%$ ) were found for all compounds (Table 2). Comparing the prediction errors using the non-linear model and the LSS-model, it is clear that the prediction accuracy can be significantly improved. Moreover, the prediction errors using the non-linear model in Table 3 were much better compared to the average prediction errors in Table 2 . The latter could be explained by the difference in gradient range between the best possible solution (94-90.6\%ACN) and the gradient test runs (95-85 and $92-85 \% \mathrm{ACN}$ ).

We also compared the calculated (experimental) $R_{S}=\left(t_{R, 2}-t_{R, 1}\right) /\left(w_{1}+w_{2}\right)$ and the predicted $R_{S}$ using Eq. (8) (Fig. 4). A similar trend in experimental and predicted $R_{S}$ was found, immediately indicating the critical pairs, although the absolute values differed significantly for the later eluting compounds.

\subsubsection{Coupled columns}

Since none of the considered columns could provide sufficient separation of all compounds, we investigated the possibilities to 


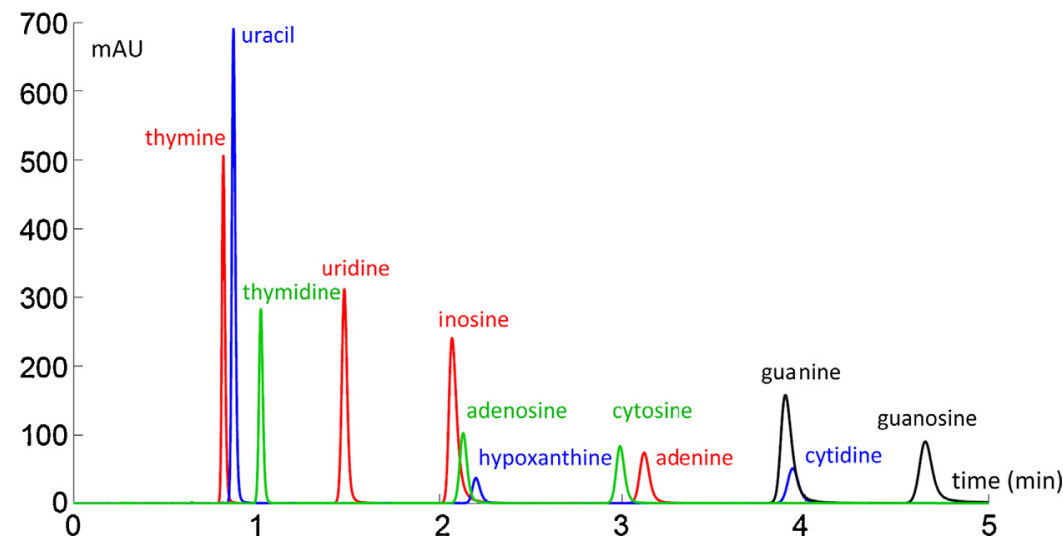

Fig. 3. Overlay chromatogram of 12 nucleobases and nucleosides on the pentahydroxy stationary phase at pH=6. Gradient conditions: $94-90.6 \% A C N$ in 3.36 min.

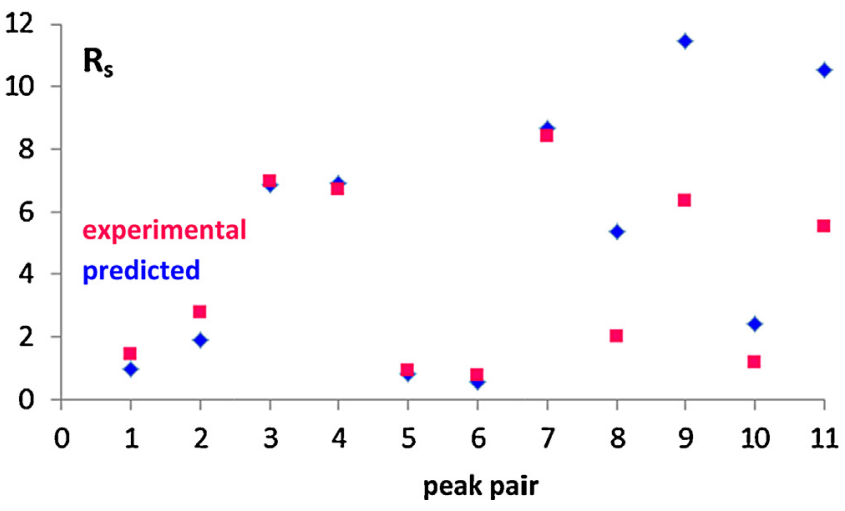

Fig. 4. Experimental and predicted $R_{s}$ using Eq. (8) for chromatogram in Fig. 3.

perform gradient prediction using coupled columns with different stationary phase chemistries to optimize the separation.

Using a coupled-column system, gradient elution retention time predictions can still be done using analytical expressions. The fundamental gradient equation is now a sum of two integrals, using both retention models:

$$
\begin{aligned}
t_{0}= & \int_{0}^{t_{R}-t_{0}} \frac{d t_{s}}{k(\varphi)}=\int_{\varphi_{0, \text { column } 1}}^{\varphi_{\text {elution,column } 1}} \frac{d \varphi}{k^{\text {column } 1}(\varphi)} \\
& +\int_{\varphi_{0, \text { column } 2}=\varphi_{\text {elution, column } 1}}^{\varphi_{\text {elution, column } 2}} \frac{d \varphi}{k^{\text {column } 2}(\varphi)}
\end{aligned}
$$

where the first and the second integrals describe the part of the gradient felt by the compound in the first and the second column, respectively. The first integral includes the dwell time of the system $t_{D}$, the column dead time $t_{0}$ and the retention parameters $k_{w}^{\prime}, S_{1}$ and $S_{2}$ from the first column to calculate the fraction of ACN at elu-

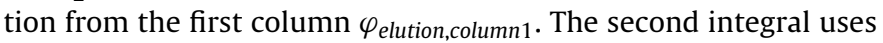
$\varphi_{\text {elution,column } 1}$ as the start of the gradient $\left(\varphi_{0, \text { column } 2}=\varphi_{\text {elution,column } 1)}\right.$ and includes a zero dwell time, the column dead time $t_{0}$ and the retention parameters $k_{w}^{\prime}, S_{1}$ and $S_{2}$ from the second column. The expression for $\varphi_{\text {elution }}$ is:

$\phi_{\text {elution }}=\frac{\phi_{0}+\left[1+S_{2} \phi_{0}\right] / S_{1} \ln \left(1+\beta k_{w}^{\prime} S_{1}\left(t_{0}-t_{D} / k_{0}\right) \exp \left(-S_{1} \phi_{0} /\left(1+S_{2} \phi_{0}\right)\right)\right)}{1-\left[S_{2}\left(1+S_{2} \phi_{0}\right)\right] / S_{1} \ln \left(1+\beta k_{w}^{\prime} S_{1}\left(t_{0}-t_{D} / k_{0}\right) \exp \left(-S_{1} \phi_{0} / 1+S_{2} \phi_{0}\right)\right)}$

where $\varphi_{0}$ is respectively $\varphi_{0, \text { column } 1}$ and $\varphi_{0 \text {,column } 2}$. From $\varphi_{\text {elution }}$ the retention time $t_{R}$ in both columns can be calculated:

$\phi_{\text {elution }}=\phi_{0}+\beta\left(t_{S}-t_{D}\right)=\phi_{0}+\beta\left(t_{R}-t_{0}-t_{D}\right) \Leftrightarrow t_{R}=t_{D}+t_{0}+\frac{\phi_{\text {elution }}-\phi_{0}}{\beta}$
Then, the total retention time is the sum of both individual retention times. The resulting analytical expression for the gradient retention factor is:

$k_{\text {eff }}=\frac{\left(t_{R, \text { column } 1}+t_{R, \text { column } 1}\right)-\left(t_{0, \text { column } 1}+t_{0, \text { column } 2}\right)}{\left(t_{0, \text { column } 1}+t_{0, \text { column } 2}\right)}$

The computer grid search was extended to the coupled column systems and a combination of the amide and the bare silica stationary phases was proposed by the algorithm, as the elution order was similar on both columns (elution order of adenosine and hypoxanthine was reversed compared to the pentahydroxy column, see Fig. 1) directing toward better separation when coupling both columns in series compared to the single column system. Almost baseline separation was obtained for the twelve nucleobases and nucleosides in less than $7 \mathrm{~min}$ (compared to $55 \mathrm{~min}$ from Massolini and coworkers [22]). It must be noted that full baseline separation could possibly be obtained by increasing the column length (and the corresponding analysis time).

The prediction errors using the coupled columns were somewhat worse than for the single-column system (Table 4), which may be due to the tubing that was used to couple the columns, but (almost) baseline separation was obtained for all twelve compounds (Fig. 5). The worst predicted compounds for the optimized gradient on the coupled column system were hypoxanthine and adenosine. However, no good reason was found for this behavior as none of these two compounds is the most ionized and the number of $\mathrm{H}$-bond donor or acceptor groups is not larger than for the other test molecules. Therefore, no stronger interactions exist between these two compounds and the stationary phase than for the other compounds.

Also for the coupled columns, we compared the experimental and the predicted $R_{S}$ (Eq. (8)). As for the single column system, a

Table 4

Retention time predictions ( $\mathrm{min}$ ) using two coupled columns (amide and bare silica stationary phase). Gradient conditions: $95-85 \% \mathrm{ACN}$ in $6 \mathrm{~min}$.

\begin{tabular}{llll}
\hline & $t_{R, \text { predicted }}(\min )$ & $t_{R, \text { experiment }}(\min )$ & \%error \\
\hline Thymine & 1.773 & 1.804 & $-1.7 \%$ \\
Uracil & 1.944 & 1.971 & $-1.4 \%$ \\
Thymidine & 2.177 & 2.212 & $-1.6 \%$ \\
Uridine & 3.185 & 3.205 & $-0.6 \%$ \\
Inosine & 3.693 & 3.774 & $-2.2 \%$ \\
Hypoxanthine & 3.761 & 3.918 & $-4.0 \%$ \\
Adenosine & 3.854 & 4.071 & $-5.3 \%$ \\
Cytosine & 4.764 & 4.731 & $0.7 \%$ \\
Adenine & 4.944 & 4.921 & $0.5 \%$ \\
Guanine & 5.284 & 5.354 & $-1.3 \%$ \\
Cytidine & 5.628 & 5.673 & $-0.8 \%$ \\
Guanosine & 6.177 & 6.271 & $-1.5 \%$ \\
\hline
\end{tabular}




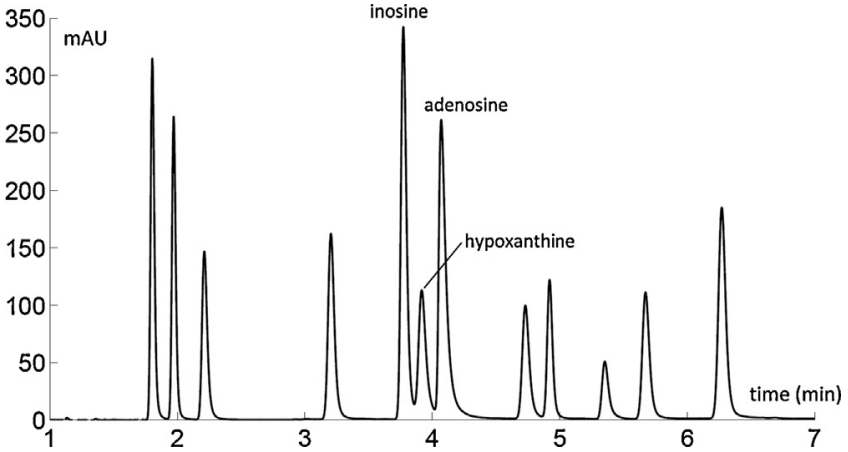

Fig. 5. Separation of twelve nucleobases and nucleosides using two coupled columns (amide and bare silica stationary phase). Gradient conditions: 95-85\%ACN in $6 \mathrm{~min}$.

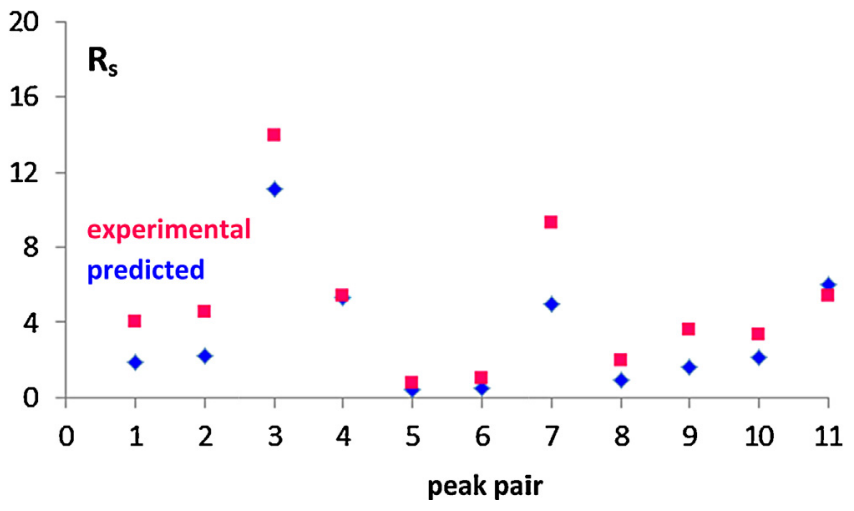

Fig. 6. Experimental and predicted $R_{S}$ using Eq. (8) for chromatogram in Fig. 5.

similar trend in $R_{S}$ was found for all peaks, indicating the critical pairs (Fig. 6). This again verifies the use of individual peak modeling, including retention time and resolution, using coupled column systems to optimize the gradient conditions for HILIC separations. However, the absolute prediction errors were still significant. Therefore, attempts to include these $R_{S}$ predictions will be included in future research.

The influence of the columns order was also investigated and no significant difference in selectivity and $R_{s, c r i t}$ was observed in this case.

\section{Conclusions}

In this study, the separation of twelve nucleobases and nucleosides was optimized via chromatogram simulation, i.e., prediction of individual retention times and estimation of the peak widths, with the use of the empirical (reversed-phase) non-linear model proposed by Neue and Kuss [21]. As none of the tested single columns could resolve all peaks, the modeling was extended to coupled-column systems (with different stationary phase chemistries) and analytical expressions were derived. The optimized gradient using a combination of an amide and a bare silica stationary phase and obtained via the adopted modeling and computer-optimization approach resulted in almost baseline separation of the twelve nucleobases and nucleosides with an analysis time of less than $7 \mathrm{~min}$. The optimized separation conditions were obtained in less than $4 \mathrm{~h}$ of instrument time (including equilibration times of 4 min corresponding to roughly 20 column volumes). As such, this study provides an example of a case where individual retention modeling and computer-optimization can be used as a way to optimize the gradient conditions in the HILIC mode provided a non-linear model such as the Neue and Kuss model can be used. For compounds whose retention mechanism is more complex than that of the presently studied set, search approaches depending less strongly on an accurate retention modeling, such as the predictive elution window shifting and stretching (PEWS ${ }^{2}$ ) method, should be preferred to optimize the gradient conditions in the HILIC separation mode $[11,12]$.

Limitations of the individual peak modeling approach include the retention time robustness due to phase aging, inter-/intra-day method reproducibility and sample matrix effects. In worst case, this may require the prediction to be redone in some cases (e.g. batch-batch reproducibility of columns will need consideration). On the other hand, the proposed optimization algorithm for HILIC separations can easily be integrated in commercial reversed-phase software such as Drylab [26], including a robustness module, to determine by which factors the critical resolution $R_{s, c r i t}$ is influenced mostly.

\section{Acknowledgments}

Dave Bell is gratefully acknowledged for providing the Supelco columns. The authors acknowledge the financial support from the Research Foundation Flanders (FWO-Vlaanderen). E.T. is the recipient of a PhD-fellowship (FWO-Vlaanderen).

\section{References}

[1] T. Jupille, L. Snyder, MolnarF I., Optimizing multi-linear gradients in HPLC, LC GC Europe (2002) 596-601.

[2] V. Concha-Herrara, G. Vivó-Truyols, J.R. Torres-Lapasio, M.C. García-AlvarezCoque, Limits of multi-linear gradient optimization in reversed-phase liquid chromatography, J. Chromatogr. A 1063 (2005) 79-88.

[3] P. Nikitas, A. Pappa-Louisi, K. Papachristos, Optimisation technique for stepwise gradient elution in reversed-phase liquid chromatography, J. Chromatogr. A 1033 (2004) 283-289.

[4] M. De Beer, F. Lynen, M. Hanna-Brown, P. Sandra, Multiple step gradient analysis in stationary phase optimised selectivity LC for the analysis of complex mixtures, Chromatographia 69 (2009) 609-614.

[5] P. Jandera, J. Churacek, Gradient elution in column liquid chromatography: theory and practice, J. Chromatogr. 170 (1979) 1-10.

[6] Y. Shan, Z. Weibing, A. Seidel-Morgenstern, R. Zhao, Y. Zhang, Multi-segment linear gradient optimization strategy based on resolution map in HPLC, Sci. China Ser. B: Chem. 46 (2006) 315-325.

[7] J.C. Berridge, Simplex optimization of high-performance liquid chromatographic separations, J. Chromatogr. 485 (1989) 3-14.

[8] M. Lämmerhofer, P.D. Di Eugenio, I. Molnar, W. Lindner, Computerized optimization of the high-performance liquid chromatographic enantioseparation of a mixture of 4-dinitrophenyl amino acids on a quinine carbamatetype chiral stationary phase using Drylab, J. Chromatogr. B 689 (1997) $123-135$.

[9] E.F. Hewitt, P. Lukulay, S. Galushko, Implementation of a rapid and automated high performance liquid chromatography method development strategy for pharmaceutical drug candidates, J. Chromatogr. A 1107 (2006) $79-87$.

[10] B. Debrus, P. Lebrun, E. Rozet, T. Schofield, J.K. Mbinze, R.D. Marini, S. Rudaz, B. Boulanger, Ph. Hubert, A new method for quality by design robust method optimization in liquid chromatography, LC-GC Europe 26 (2013) 370-375.

[11] E. Tyteca, A. Liekens, D. Clicq, A. Fanigliulo, B. Debrus, S. Rudaz, D. Guillarme, G Desmet, Predictive elution window shifting and stretching as a generic search strategy for automated method development for liquid chromatography, Anal. Chem. 84 (2012) 7823-7830.

[12] E. Tyteca, A. Periat, S. Rudaz, G. Desmet, D. Guillarme, Retention modeling and method development in hydrophilic interaction chromatography, J. Chromatogr. A 1337 (2014) 116-127.

[13] D. McCalley, Study of the selectivity, retention mechanisms and performance of alternative silica-based stationary phases for separation of ionised solutes in hydrophilic interaction chromatography, J. Chromatogr. A 1217 (2010) 3408-3417.

[14] G. Greco, S. Grosse, T. Letzel, Study of the retention behavior in zwitterionic hydrophilic interaction chromatography of isomeric hydroxy- and aminobenzoic acids, J. Chromatogr. A 1235 (2012) 60-67.

[15] G. Jin, Z. Guo, F. Zhang, X. Xue, Y. Jin, X. Liang, Study on the retention equation in hydrophilic interaction liquid chromatography, Talanta 76 (2008) $522-527$.

[16] A. Karatapanis, Y.C. Fiamegos, C.D. Stalikas, A revisit to the retention mechanism of hydrophilic interaction liquid chromatography using model organic compounds, J. Chromatogr. A 1218 (2008) 2871-2879. 
[17] P. Jandera, T. Hájek, Utilization of dual retention mechanism on columns with bonded PEG and diol stationary phases for adjusting the separation selectivity of phenolic and flavone natural antioxidants, J. Sep. Sci. 32 (2009) 3603-3619.

[18] J.W. Dolan, D.C. Lommen, L.R. Snyder, High-performance liquid chromatographic computer simulation based on a restricted multi-parameter approach: I. Theory and verification, J. Chromatogr. 535 (1990) 55-74.

[19] P. Jandera, Can the theory of gradient liquid chromatography be useful in solving practical problems? J. Chromatogr. A 1126 (2006) 195-218.

[20] P. Nikitas, A. Pappa-Louisi, New approaches to linear gradient elution used for optimization in reversed-phase liquid chromatography, J. Liq. Chromatogr. Relat. Technol. 32 (2009) 1527-1576.

[21] U.D. Neue, H.-J. Kuss, Improved reversed-phase gradient retention modeling, J. Chromatogr. A 1217 (2010) 3794-3803.

[22] L.R. Snyder, D.L. Saunders, Optimized solvent programming for separations of complex samples by liquid-solid adsorption chromatography in columns, J. Chromatogr. Sci. 7 (1969) 195-208.
[23] G. Marrubini, B.E. Castillo Mendoza, G. Massolini, Separation of purine and pyrimidine bases and nucleosides by hydrophilic interaction chromatography, J. Sep. Sci. 33 (2010) 803-816.

[24] P. Nikitas, A. Pappa-Louisi, P. Agrafiotou, A. Mansour, Multilinear gradient elution optimization in reversed-phase liquid chromatography based on logarithmic retention models: application to separation of a set of pyrines, pyrimidines and nucleosides, J. Chromatogr. A 1218 (2011) $5658-5663$.

[25] B. Debrus, P. Lebruna, A. Ceccat, G. Caliaroc, E. Rozet, I. Nistor, R. Opreand, F.J. Rupérez, C. Barbas, B. Boulanger, P. Hubert, Application of new methodologies based on design of experiments, independent component analysis and design space for robust optimization in liquid chromatography, Anal. Chim. Acta 691 (2011) 33-42.

[26] A.H. Schmidt, I. Molnar, Using an innovative quality-by-design approach for development of a stability indicating UHPLC method for ebastine in the API and pharmaceutical formulations, J. Chromatogr. A 78-79 (2013) 65-74. 